\title{
Two new species of Tynanthus Miers (Bignonieae, Bignoniaceae) from Brazil
}

\author{
Maria Cláudia M. P. de Medeiros', Lúcia G. Lohmann' \\ I Universidade de São Paulo, Instituto de Biociências, Departamento de Botânica, Rua do Matão, 277, \\ 05508-090, São Paulo, SP, Brazil \\ Corresponding authors: Maria Cláudia M. P. de Medeiros (mariaclaudiamedeiros@hotmail.com);
}

Lúcia G. Lohmann (llohmann@usp.br)

Academic editor: Alan Paton | Received 7 July 2014 | Accepted 7 October 2014 | Published 24 October 2014

Citation: Medeiros MCMP, Lohmann LG (2014) Two new species of Tynanthus Miers (Bignonieae, Bignoniaceae) from Brazil. PhytoKeys 42: 77-85. doi: 10.3897/phytokeys.42.8210

\begin{abstract}
Tynanthus is a genus of lianas that is broadly distributed through the Neotropics. Two new species of Tynanthus from Brazil are here described and illustrated: T. densiflorus, from Amazonas, and T. espiritosantensis, from Espírito Santo. T. densiflorus is recognized by the conspicuous interpetiolar glandular fields, a feature rarely found in Tynanthus, and the dense thyrses. Tynanthus espiritosantensis, on the other hand, is recognized by the bromeliad-like prophylls of the axillary buds and the lax thyrses. Information on the distribution, conservation status and morphologically similar species are provided.
\end{abstract}

\section{Keywords}

Amazonia, Atlantic forest, "cipó-cravo", lianas, neotropical flora

\section{Introduction}

Tynanthus Miers (Bignonieae, Bignoniaceae) is a monophyletic genus of lianas that is easily recognized by small bilabiate flowers, fruits with raised margins and by the smell of cloves in vegetative organs (Lohmann 2006). These features, along with flowers arranged in thyrses, corolla externally densely pubescent, thecae curved forward, ovary densely pubescent and poorly developed nectar disk characterize the genus (Medeiros and Lohmann submitted). Species of Tynanthus are distributed throughout the Neotropics, occurring predominantly in wet forests (Lohmann and Taylor 2014). The 
highest diversity of Tynanthus is found in Brazil, with most species occurring in Amazonia and the Atlantic Forest.

During the preparation of a taxonomic revision of Tynanthus (Medeiros and Lohmann submitted), multiple collections with morphological features that did not match any of the described species were found. Additional fieldwork and molecular phylogenetic studies provided further support for the recognition of two new species in the genus, one from the Amazon region and another from the Atlantic Forest of Brazil.

\section{Material and methods}

Morphological analyses and compilation of phenology and distribution data were based on specimens deposited at CVRD, G, INPA, K, MBM, MG, MO, NY, RB, SP, SPF and US (Thiers, continuously updated). Descriptions were elaborated following terminology presented in Radford (1986), Weberling (1989), the Leaf Architecture Working Group (1999), Gomes-Silva (2009), Nogueira et al. (2013) and Lohmann and Taylor (2014).

Key to the species of Tynanthus with known occurrences in Brazil

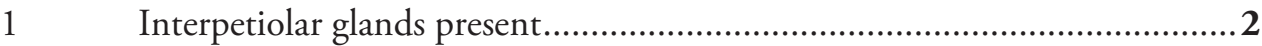

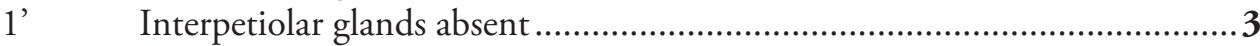

2 Leaflets with caudate-mucronate apices; flowers arranged in dense thyrses....

T. densiflorus

2' Leaflets with acuminate or obtuse-mucronate apices; flowers arranged in lax thyrses

T. pubescens

3 Prophylls of the axillary buds foliaceous.................................................. 4

3' Prophylls of the axillary buds minute and triangular, or bromeliad-like ......5

4 Young branchlets puberulent to glabrescent; tendrils trifid; corolla 1.2-1.7 $\mathrm{cm}$

T. panurensis

4' Young branchlets villous to pubescent; tendrils simple; corolla $0.4-0.8 \mathrm{~cm}$..

$5 \quad$ Prophylls of the axillary buds minute and triangular..............u.u. 6

5 $\quad$ Prophylls of the axillary buds bromeliad-like ..............................................

6 Flowers arranged in dense thyrses; calyx laciniate ...................T. fasciculatus

6) Flowers arranged in lax thyrses; calyx minutely denticulate or truncate.......8

7 Leaflet domatia with trichomes; petioles, petiolules and inflorescence axis without patelliform trichomes........................................ T.espiritosantensis

7' Leaflet domatia without trichomes; petioles, petiolules and inflorescence axis with patelliform trichomes............................................. T. schumannianus

8 Branchlets tomentose to pubescent throughout; fruits unwinged, with margins slightly raised 
8' Branchlets glabrescent (sometimes pubescent only at the nodes); fruits winged, with margins prominently raised ...................................................

9 Leaflets with acuminate-mucronate apices; corolla $1-1.4 \mathrm{~cm}$...... T. labiatus

9' Leaflets with caudate-mucronate apices; corolla $0.5-0.9 \mathrm{~cm} .$. T. micranthus

\section{Taxonomy}

\section{Tynanthus densiflorus M.C. Medeiros \& L.G. Lohmann, sp. nov. urn:lsid:ipni.org:names:77142875-1}

Type. Brazil. Amazonas: Manaus-Itacoatiara, km 26, Reserva Florestal Adolpho Ducke, 16 Aug 1996, L.C. Procópio et al. 14 (holotype: INPA-189631!; isotypes: G!, K!, MG, MO!, NY!, RB!, SP!, U) Fig. 1A-I.

Diagnosis. Tynanthus densiflorus differs from Tynanthus panurensis (Bureau) Sandwith by the interpetiolar gland fields (lacking in $T$. panurensis), minute triangular prophylls of the axillary buds (versus foliaceous in $T$. panurensis) and dense thyrses (versus lax in T. panurensis). It further differs from Tynanthus pubescens A.H. Gentry in the leaflets with a caudate-mucronate apex (versus acuminate or obtuse-mucronate apices in T. pubescens) and the dense inflorescences (versus lax in T. pubescens).

Description. Liana. Branchlets subtetragonal to terete, finely striate, with lenticels, pubescent to puberulent, with simple and peltate trichomes; interpetiolar ridge absent or present; interpetiolar glands present; prophylls of the axillary buds $0.5-0.8 \mathrm{~mm}$ long, 1-2.5 mm wide, minute, shallowly triangular, puberulent throughout, with simple and peltate trichomes. Leaves $2-3$ foliolate; terminal leaflets modified into a trifid tendril; petioles and petiolules with a more or less conspicuous canalicule on the upper side, puberulent to glabrescent throughout, with simple and peltate trichomes; petioles $1.8-5.6 \mathrm{~cm}$ long; petiolules (0.6-)1.4-3.8 cm long, lateral ones with equal lengths and the terminal one longer, when present; leaflets (3.2-)5-16.1 cm long, (1.3-)2-9.5 cm wide, membranous to chartaceous (sometimes subcoriaceous), discolor or concolor, ovate, apex caudate, mucronate, base cuneate to truncate or subcordate, symmetrical or asymmetrical, margin entire; the abaxial surface pubescent to puberulent throughout (sometimes only on and near the veins), with simple, peltate and patelliform trichomes; the adaxial surface pubescent to glabrescent throughout (sometimes only on and near the veins), with simple, peltate and patelliform trichomes; glandular trichomes evenly distributed throughout both surfaces; first venation pinnate, second venation weak brochidodromous, third venation alternate percurrent (sometimes random reticulate); pocket domatia with (sometimes without) trichomes. Inflorescence $3-9.5 \mathrm{~cm}$ long, a thyrse, axillary, dense, corymbose to conical in aspect; axis densely pubescent to puberulent, with simple and peltate trichomes; inflorescence bracts $0.5-2.5 \mathrm{~mm}$ long, predominantly caducous, triangular to linear triangular, densely pubescent to pubescent throughout; floral bracts $0.4-0.6 \mathrm{~mm}$ long, triangular; floral pedicels 1-7 $\mathrm{mm}$ long. Calyx green to grayish, 1.5-2.2 mm long, 1.4-1.9 mm wide, membranous 


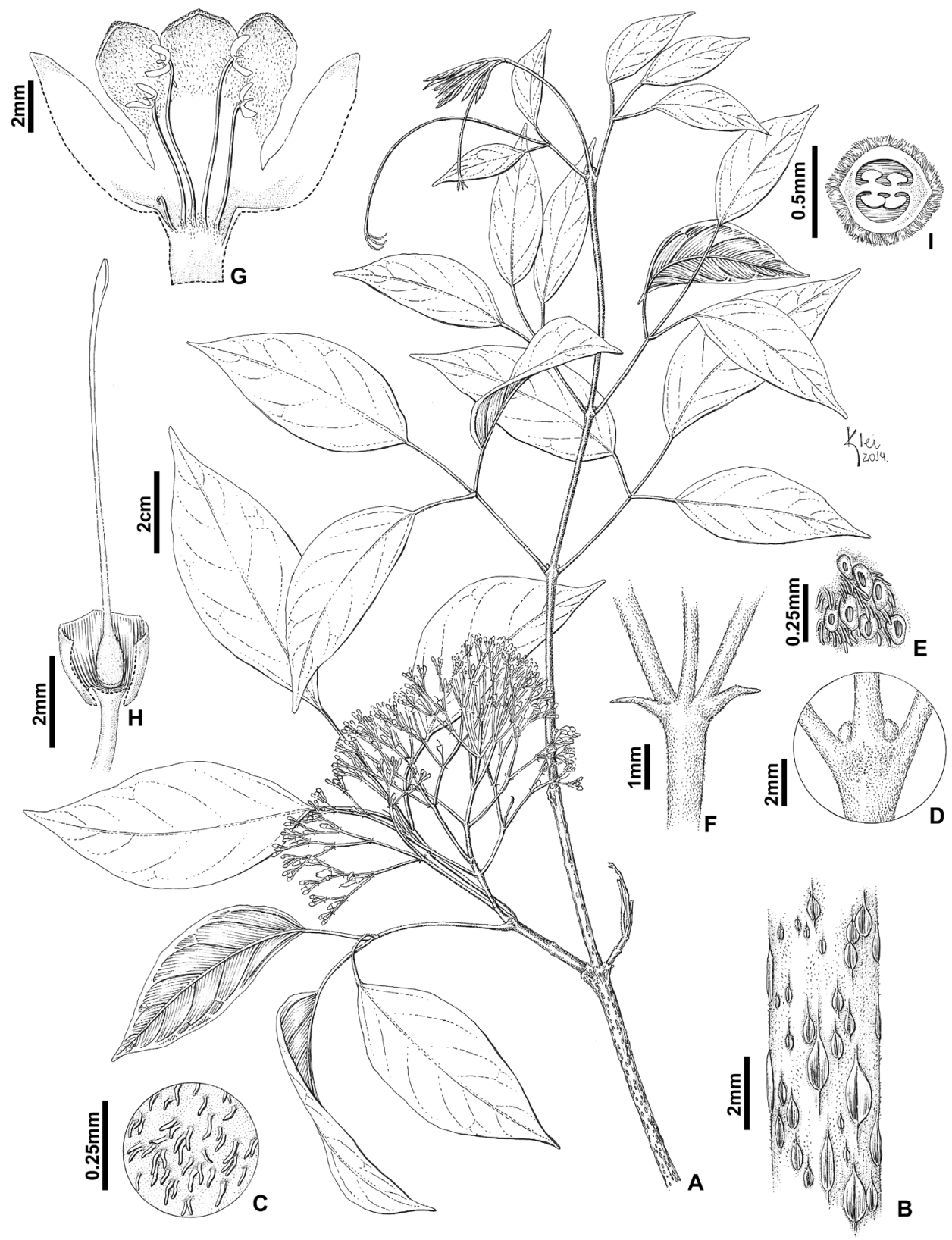

Figure I. Tynanthus densiflorus sp. nov.: A Flowering branch B Detail of lenticels in the oldest portion of branchelet $\mathbf{C}$ Detail of pubescent indumentum in the youngest portion of branchelet D-E Interpetiolar glands $\mathbf{F}$ Detail of inflorescence axis with bracts $\mathbf{G}$ Open corolla showing the androecium $\mathbf{H}$ Open calyx showing the gynoecium I Ovary cross section showing ovules [L.C. Procópio 14 (NY)].

to chartaceous, with a transversal aperture, truncate or minutely 5-denticulate, densely pubescent to pubescent outside, with simple and peltate trichomes, glabrous inside; lobes $0.1-0.2 \mathrm{~mm}$ long. Corolla cream or pale yellow, $0.8-1.5 \mathrm{~cm}$ long, $0.3-0.5 \mathrm{~cm}$ 
wide at the tube opening, bilabiate, with two (almost totally fused) upper lobes and three lower lobes, densely pubescent throughout outside, with simple and peltate trichomes; tube 3-5 mm long, internally glabrous at the top, tomentose at the base, with simple, long and short stipitate trichomes; nectar guides present, yellow; lobes entire, densely pubescent to pubescent throughout lower ones and at margins of or throughout upper ones; upper ones $0.4-1.4(-2.9) \mathrm{mm}$ long, $0.7-1.5(-2.4) \mathrm{mm}$ wide, acute to obtuse; lower ones 2.1-4 mm long, 2-3.6 mm wide, obtuse to rounded. Androecium with four fertile stamens inserted at $1.5-2.5 \mathrm{~mm}$ from the base of the corolla; shorter ones 3.5-5.5 mm long; longer ones 4.5-7 mm long; filaments with long and short stipitate trichomes at the base; anther thecae cream, 1.1-1.4 mm long, obovate to elliptic, divergent and reflexed forward, glabrous, subexserted; connective extending $0.2-0.3 \mathrm{~mm}$ beyond anther attachment; staminode covered with long and short stipitate trichomes, $1.5-2.7 \mathrm{~mm}$ long. Gynoecium ca. 7-9 mm long; ovary $1.3-1.5 \mathrm{~mm}$ long, $0.7-0.8 \mathrm{~mm}$ wide, conical, velutinous, with simple trichomes, with a ring of longer trichomes at the base, with two or four series of ovules per locule; nectar disc reduced, not evident; style 5-7 mm long, tomentose at the base, with simple trichomes; stigma with lamellae lanceolate, glabrous. Fruit not seen. Seeds not seen.

Distribution and habitat. Known from wet forests in Manaus and proximity (Amazonas, Brazil).

Etymology. The species epithet refers to the thyrses with flowers densely arranged.

Phenology. Flowers in August. Fruiting period is unknown.

Conservation status. According to IUCN (2001) criteria, this species is considered Vulnerable (VU B2ab(iii)). The type collection is from a protected area (Reserva Florestal Adolpho Ducke), where a reasonable number of individuals are found (pers. obs.). Nevertheless, the proximity of Manaus can be considered a region characterized by continuous urbanization. Additional studies on distribution and abundance of $T$. densiflorus are still necessary in order to confirm its conservation status.

Discussion. Tynanthus densiflorus is characterized by dense thyrses, with a corymbose to conical aspect, as well as the presence of interpetiolar glands. The type collection of $T$. densiflorus was treated as T. panurensis (Bureau) Sandwith for the Guide of the Ducke Reserve (Lohmann and Hopkins 1999). Indeed, the two species are similar in the occurrence of ovate leaflets and corolla tube that is internally tomentose at base. However, the presence of interpetiolar glands in $T$. densiflorus (versus absent in $T$. panurensis), the minute prophylls (versus foliaceous in T. panurensis) and dense inflorescences (versus lax in T. panurensis) allow the distinction of these taxa. Tynanthus pubescens A.H. Gentry is another species that is morphologically similar to T. densiflorus. Both taxa have similar corolla lengths (around 1-1.6 cm in T. pubescens) and show interpetiolar gland fields; however, the caudate-mucronate leaflet apex (versus acuminate or obtuse-mucronate in $T$. pubescens) and the dense inflorescences (versus lax in $T$. pubescens) differentiate both taxa.

Additional specimens examined. BRAZIL. Amazonas: $2-5 \mathrm{~km} \mathrm{~N}$ of ManausItacoatiara Road at km 79 near Rio Preto da Eva, 100-200 m, 24 November 1974, A. Gentry 12849 (INPA, MG, MO). Rio Camanau, 28 June 1987, P. Grenand et al. 2787 (INPA). Manaus, Campus of INPA, Estrada do Aleixo, 22 November 1974, 
A. Gentry 12792 (INPA); 30 November 1974, A. Gentry 13018 (INPA, MO); Ibid., Transect vouchers, Line 1, 11 December 1974, A. Gentry 13181 (INPA, MO); Estrada do Aleixo near Manaus, km 6-7 past INPA, 2 December 1974, A. Gentry 13040 (INPA, MO); Reserva Florestal Adolpho Ducke, Parcela PPBio (L03 1000 m), 100 m, 02 56'03"S, 5957'32"W, 14 December 2010, M.C. Medeiros et al. 21 (SPF); Ibid., próximo à estação meteorológica, $120 \mathrm{~m}, 02^{\circ} 55^{\prime} 37^{\prime \prime S}, 59^{\circ} 58^{\prime} 33^{\prime \prime W}, 15$ December 2010, M.C. Medeiros et al. 22 (SPF); Ibid., proximidades do refeitório da base da reserva, na beira da estrada, $110 \mathrm{~m}, 02^{\circ} 55^{\prime} 59^{\prime \prime S}$, 5957'56"W, 16 December 2010, M.C. Medeiros et al. 25 (SPF).

\section{Tynanthus espiritosantensis M.C. Medeiros \& L.G. Lohmann, sp. nov. urn:Isid:ipni.org:names:77142876-1}

Type. Brazil. Espírito Santo: Linhares, Reserva Natural da CVRD, Estrada Oiticica, km 2.3, 6 Feb 2008, D.A. Folli 5931 (holotype: CVRD-11073!; isotype: SPF!) Fig. 2A-I.

Diagnosis. Tynanthus espiritosantensis differs from Tynanthus schumannianus (Kuntze) A.H. Gentry by the presence of trichomes in the leaflet domatia (versus absence in T. schumannianus), lack of patelliform trichomes on petioles, petiolules and inflorescence axis (versus presence in T. schumannianus) and larger calyx, $2.3-2.7 \mathrm{~mm}$ long, 1.8-2.5 mm wide (versus 1-2 mm long, 1.1-1.9 mm wide in T. schumannianus).

Description. Liana. Branchlets tetragonal to terete, finely striate, with lenticels, glabrescent (sometimes pubescent at the nodes), with peltate and patelliform trichomes (sometimes simple trichomes also present); interpetiolar ridge absent; interpetiolar glands absent; prophylls of the axillary buds $1.2-2.5 \mathrm{~mm}$ long, $0.7-1.1 \mathrm{~mm}$ wide, bromeliad-like, glabrescent (rarely puberulent), with peltate trichomes (rarely with simple trichomes as well). Leaves (2-)3 foliolate; terminal leaflets modified into a trifid tendril; petioles and petiolules with a more or less conspicuous canalicule on the upper side, puberulent throughout, with simple and peltate trichomes; petioles $1-6 \mathrm{~cm}$ long; petiolules $0.5-3.5 \mathrm{~cm}$ long, lateral ones with equal lengths and the terminal one longer, when present; leaflets (4-)5-11.9 cm long, (1.5-)1.9-5.4 cm wide, membranous to chartaceous, discolor, elliptic, apex acuminate or caudate, mucronate, base cuneate, symmetrical, margin entire; the abaxial surface glabrescent (sometimes pubescent) on and near the veins, with peltate and patelliform trichomes (sometimes also simple); the adaxial surface glabrescent on and near the veins, with peltate and patelliform trichomes; glandular trichomes distributed especially on the abaxial surface; first venation pinnate, second venation weak brochidodromous, third venation alternate percurrent (sometimes random reticulate); pocket domatia with trichomes. Inflorescence 3.6-7 cm long, a thyrse, axillary, lax, conical in aspect; axis pubescent, with simple and peltate trichomes; inflorescence bracts $0.7-3.9(-9) \mathrm{mm}$ long, predominantly caducous, triangular to linear triangular, pubescent throughout or only at margins; floral bracts $0.5-0.7$ $\mathrm{mm}$ long, triangular; floral pedicels $3.5-9 \mathrm{~mm}$ long. Calyx green, $2.3-2.7 \mathrm{~mm}$ 


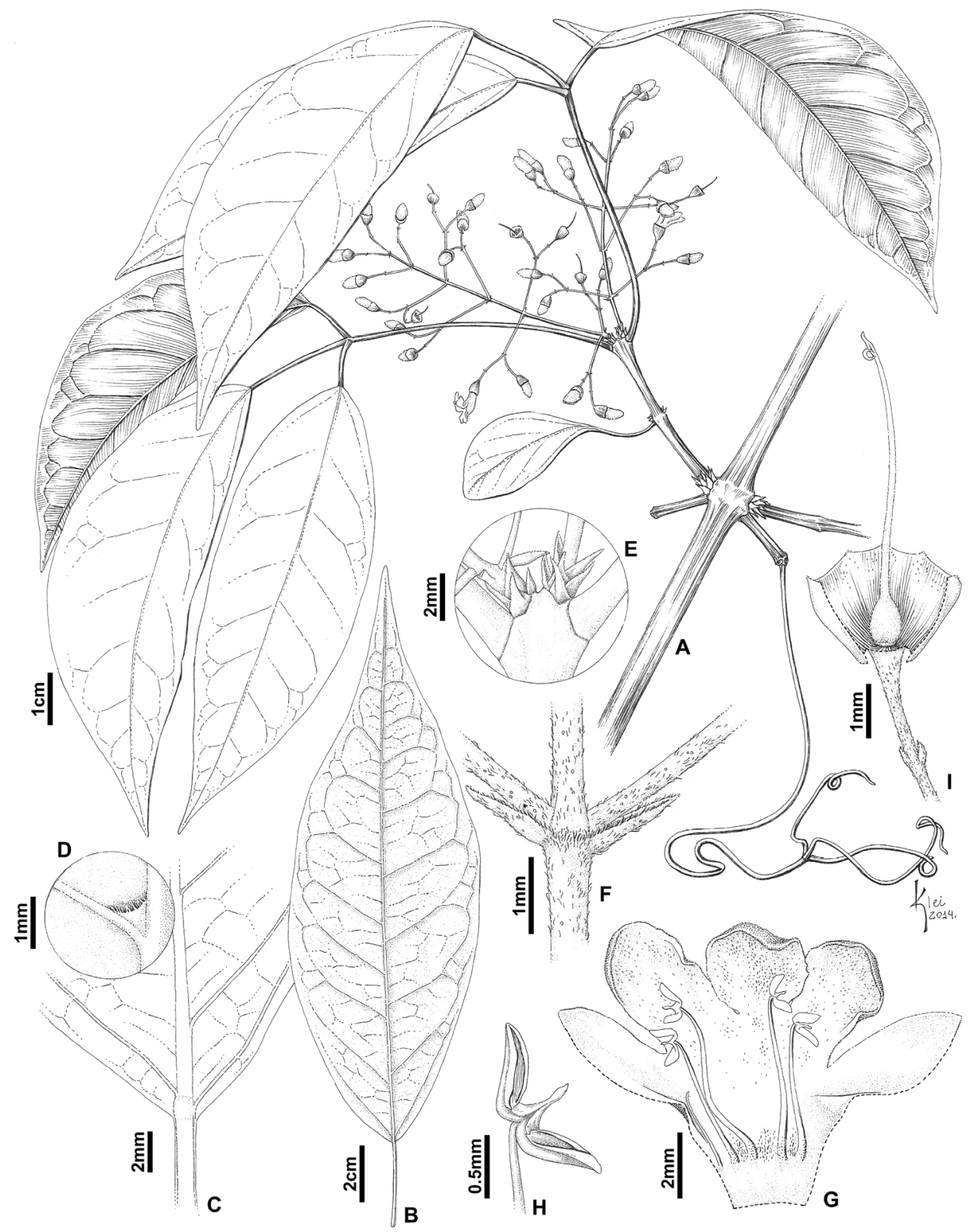

Figure 2. Tynanthus espiritosantensis sp. nov.: A Flowering branch B-D Leaflet with pubescent domatia in the abaxial surface $\mathbf{E}$ Interpetiolar region with bromeliad-like prophylls of the axillary buds $\mathbf{F}$ Detail the of inflorescence axis, showing bracts, simple and peltate trichomes $\mathbf{G}$ Open corolla, showing the androecium H Anther I Open calyx showing the gynoecium [D.A. Folli 5931 (SPF)].

long, 1.8-2.5 mm wide, membranous to chartaceous, with a transversal (sometimes oblique) aperture, minutely 5-denticulate, glabrescent (sometimes pubescent at teeth) outside, with simple, peltate and patelliform trichomes, glabrous inside; 
lobes $0.1-0.4 \mathrm{~mm}$ long. Corolla white, $0.7-0.8 \mathrm{~cm}$ long, $0.25-0.34 \mathrm{~cm}$ wide at the tube opening, bilabiate, with two (almost totally fused) upper lobes and three lower lobes, densely pubescent throughout outside, with simple and peltate trichomes; tube $2.5-4 \mathrm{~mm}$ long, internally glabrous at the top, tomentose to pubescent at the base or glabrescent, with simple, long and short stipitate trichomes; nectar guides absent, but with a path of long and short stipitate trichomes; lobes entire, densely pubescent to pubescent throughout lower ones and at the margin of upper ones; upper ones $0.4-1.1 \mathrm{~mm}$ long, $1-1.5 \mathrm{~mm}$ wide, acute to obtuse; lower ones 1.8-3.2 $\mathrm{mm}$ long, $2.1-2.5 \mathrm{~mm}$ wide, obtuse to rounded (sometimes acute). Androecium with four fertile stamens, inserted at $1-1.5 \mathrm{~mm}$ from the base of the corolla; shorter ones 2.5-3.5 mm long; longer ones $4.5-5 \mathrm{~mm}$ long; filaments with long and short stipitate trichomes at the base; anthers thecae cream, $0.8-1.1 \mathrm{~mm}$ long, obovate to elliptic, divergent and reflexed forward, glabrous, subexserted; conective extending $0.2-0.3 \mathrm{~mm}$ beyond anther attachment; staminode glabrescent, with long and short stipitate trichomes, $2.4 \mathrm{~mm}$ long. Gynoecium ca. 4.5-6 mm long; ovary $0.8-1 \mathrm{~mm}$ long, 0.7-0.9 mm wide, conical, velutinous, with simple trichomes, with a ring of longer trichomes at the base, with two or four series of ovules per locule; nectar disc reduced, not evident; style 3.3-5 mm long, tomentose at the base, with simple trichomes; stigma with lamellae lanceolate, glabrous. Fruits not seen. Seeds not seen.

Distribution and habitat. Known exclusively from wet forests in Linhares and proximity (Espírito Santo, Brazil).

Etymology. The species epithet refers to the type locality.

Phenology. Flowers from December to February. Fruiting period is unknown.

Conservation status. According to the IUCN (2001) criteria, this species is considered Data Deficient (DD), given the small number of known collections. Further detailed investigation on the distribution of T. espiritosantensis is necessary in order to properly assess its conservation status. The two localities in which this species has been collected fall within a single municipality (Linhares), suggesting that this might represent another narrowly distributed species of Tynanthus. Fortunately, the type collection was obtained inside a protected area (Reserva Natural da CVRD).

Discussion. Tynanthus espiritosantensis is characterized by the bromeliad-like prophylls of the axillary buds and lax thyrses. This species is morphologically similar to the Amazonian T. schumannianus (Kuntze) A.H. Gentry. However, these taxa can be easily separated by the pubescent leaflet domatia (versus glabrous in T. schumannianus), absence of patelliform glands on petioles, petiolules and inflorescence axis (versus presence in T. schumannianus) and the larger calyx, 2.3-2.7 mm long, 1.8-2.5 mm wide (versus 1-2 mm long, 1.1-1.9 mm wide in T. schumannianus).

Additional specimens examined. BRAZIL. Espírito Santo: Linhares, Rancho Alto, 7 December 1984, G. Hatschbach \& J.M. Silva 48693 (MBM, MO, US); Reserva Natural da CVRD, Estrada Oiticica, próximo à porteira, antes do cruzamento com a estrada municipal, $53 \mathrm{~m}, 19^{\circ} 07^{\prime} 59^{\prime \prime} \mathrm{S}, 40^{\circ} 00^{\prime} 07^{\prime \prime} \mathrm{W}, 27$ January 2014, M.C. Medeiros \& R.B. Louzada 41 (CVRD, SPF). 


\section{Acknowledgements}

This study is part of the Ph.D. thesis of M.C.M.P.M. conducted at the Universidade de Sáo Paulo. The authors thank the Conselho Nacional de Desenvolvimento Científico e Tecnológico (CNPq) for a fellowship to M.C.M.P.M. (142379/2010-7) and a Pq-1C grant to L.G.L. (307781/2013-5); the Fundação de Amparo à Pesquisa do Estado de São Paulo (FAPESP) for a fellowship to M.C.M.P.M. (2010/51579-0), a regular research grant to L.G.L. (2011/50859-2); two collaborative Dimensions of Biodiversity-BIOTA grants supported by FAPESP, NSF, and NASA (2012/50260-6 and 2013/50297-0); and, the International Association for Plant Taxonomy for a Research Grant to M.C.M.P.M. We also thank the curators of the herbaria cited in the text for sending specimens on loan and for permission to visit and study their specimens; Instituto Nacional de Pesquisas da Amazônia (INPA) and the Reserva Natural da CVRD for authorizing us to conduct fieldwork inside the Reserves; Rafael Louzada for assistance during fieldwork; and Klei Sousa for the preparation of the botanical illustrations.

\section{References}

Gomes-Silva F (2009) Evolução de acarodomácias em Bignonieae (Bignoniaceae). MSc. Dissertation, Universidade de São Paulo, São Paulo, 114 pp.

IUCN (2001) IUCN Red List Categories and Criteria, Version 3.1. http://www.iucn.org/

Leaf Architecture Working Group (1999) Manual of leaf architecture - morphological description and categorization of dicotyledonous and net-veined monocotyledonous angiosperms. Smithsonian Institution, Washington, $65 \mathrm{pp}$.

Lohmann LG (2006) Untangling the phylogeny of Neotropical lianas (Bignonieae, Bignoniaceae). American Journal of Botany 93: 304-318. doi: 10.3732/ajb.93.2.304

Lohmann LG, Hopkins MJG (1999) Bignoniaceae. In: Ribeiro JELS, Hopkins MJG, Vicentini A, Sothers CA, Costa MAS, Brito JM, Souza MAD, Martins LHP, Lohmann LG, Assunção PACL, Pereira EC, Silva CF, Mesquita MR, Procópio LC (Eds) Flora da Reserva Ducke: Guia de Identificação das plantas vasculares de uma floresta de terra-firme na Amazônia Central. INPA/DIFD, Manaus, 608-623.

Lohmann LG, Taylor CM (2014) A new generic classification of Tribe Bignonieae (Bignoniaceae). Annals of the Missouri Botanical Garden 99: 348-489. doi: 10.3417/2003187

Nogueira A, El-Ottra JHL, Guimarães E, Machado SR, Lohmann LG (2013) Trichome structure and evolution in Neotropical lianas. Annals of Botany 112: 1331-1350. doi: $10.1093 / \mathrm{aob} / \mathrm{mct} 201$

Radford AE (1986) Fundamentals of plant systematics. Harper \& Row, New York, 512 pp.

Thiers B (continuously updated) Index Herbariorum: A global directory of public herbaria and associated staff. New York Botanical Garden's Virtual Herbarium. http://sweetgum. nybg.org/ih/ [accessed: April 2014]

Weberling F (1989) Morphology of flowers and inflorescences. Cambridge University Press, Cambridge, 348 pp. 